\section{Premasticación: una nueva forma de transmisión del virus de inmunodeficiencia humana. Primer caso pediátrico informado en Chile}

\author{
Yenis Labraña, Ana María Alvarez, Julia Villarroel y Elba Wu
}

\section{Premastication: a new way of transmitting HIV. First pediatric case reported in Chile}

The incorporation of the protocol to prevent vertical transmission (PMTCT) of HIV in pregnant women has reduced the rate of HIV transmission in children to less than $2 \%$. In Chile, currently the diagnosis of HIV infection in children is rare. Thus, one positive finding should lead us to audit compliance of the PMTCT and if this has been fully implemented should be reviewed other possible routes of HIV transmission. We present a case report that suggest that HIV can be transmitted through the consumption of foods that have been premasticated by a person infected with HIV. Premastication is a transmission path that had not been reported, being a possible explanation for some cases of late transmission of HIV in infants, so far attributed to breastfeeding. Understanding that premastication is a common behavior, you should educate people about the potential risk of transmission of diseases, including HIV, through this practice and advise HIV-infected caregivers against this practice.

Key words: HIV, Vertical Pathogen transmission, PMTCT HIV, premastication.

Palabras clave: VIH, transmisión vertical, premasticación.

\section{Introducción}

\section{L} a infección por el virus de la inmunodeficiencia humana (VIH) en niños es transmitida en más de $90 \%$ de los casos por vía vertical, durante el embarazo, parto y lactancia materna. La incorporación del protocolo de prevención de transmisión vertical (PTV) del VIH en mujeres embarazadas, que incluye el uso de fármacos anti-retrovirales en el embarazo, parto y en el recién nacido hasta las 6 semanas de vida, la indicación de cesárea, rotura prematura de membrana de menos de 4 h y suspensión de la lactancia materna, han reducido el porcentaje de transmisión del VIH en niños a menos de $2 \%$.

En Chile, actualmente el diagnóstico de infección por VIH en niños es infrecuente. Desde el año 2005 se ofrece, con consentimiento informado, el examen de anticuerpos específicos para VIH en las mujeres embarazadas, por lo que los casos confirmados inician rápidamente el protocolo de PTV. Así, al detectar un caso, se debe revisar el cumplimiento de todo el proceso del PTV y si éste se cumplió en su totalidad, deben plantearse otras vías posibles de transmisión del VIH.

Hospital San Juan de Dios, Santiago, Chile. Servicio de Pediatría, Unidad de Infectología. Recibido: 27 de septiembre de 2012 / Aceptado: 24 de enero de 2013

Correspondencia a:

Yenis Labraña

yenislabrana@gmail.com
Presentamos un caso pediátrico que nos alertó a buscar otros posibles mecanismos de transmisión y que actualmente la literatura científica ha incorporado en forma novedosa.

\section{Caso clínico}

Paciente de sexo femenino, con antecedente de madre con infección por VIH diagnosticada a las 20 sem de gestación, por lo que recibió PTV con zidovudina (AZT), lamivudina (3TC) y lopinavir/ritonavir. El parto fue por cesárea electiva y se indicó suspensión de la lactancia materna. Durante el período de RN se realizó el estudio diagnóstico de infección por VIH para niños hijos de madre con infección por VIH de acuerdo a la Norma del Instituto de Salud Pública de nuestro país. En breve, el estudio consiste en detección del genoma viral (mediante RPC) al $1^{\text {er }}$ día de vida, a los 15-30 días de nacer y a los 3 meses de edad. El estudio fue negativo para infección por VIH, dándose de alta del policlínico de Infectología a los 18 meses de vida.

A los 2 años y 4 meses presentó un cuadro de fiebre, diarrea intermitente y compromiso del estado general. Al examen físico se constató una algorra bucal extensa y adenopatías retroauriculares. Por el antecedente de ser hijo de madre con infección por VIH se solicitó serología para VIH que resultó positiva. Se inició el estudio de posibles mecanismos de transmisión diferentes al vertical. No tenía antecedentes de transfusiones, por lo que se planteó la vía sexual como la más probable. Fue evaluada por psicólogo y psiquiatra, los que no encontraron una historia sustentable. También fue evaluada por dos ginecólogos infantiles que descartaron signos de abuso sexual. Además se realizó una endosonografía rectal que fue normal, por lo que no pudo confirmarse la transmisión por vía sexual.

Reinterrogando a la madre y revisando la literatura científica reciente que describe la premasticación como una nueva forma de transmisión del VIH, la madre confirmó esta práctica en forma reiterada.

\section{Discusión}

El caso presentado concuerda con la observación que el VIH puede ser transmitido a través del consumo de alimentos que han sido premasticados por una persona infectada con el VIH.

En el año 2009 el CDC estimó que $21 \%$ de los 166 casos diagnosticados con VIH/SIDA, en niños bajo los 13 años, se debieron a otros modos de transmisión diferentes a la perinatal, incluyendo la hemofilia, transfusiones de sangre y un "factor de riesgo no reportado o no identificado".

En nuestro país, hasta diciembre de 2011 (según registros del ISP), se han descrito 344 casos de niños con infección por VIH, de los cuales 326 son hijos de madre con VIH. En los 18 casos restantes no se pudo identificar el mecanismo de transmisión.

Aunque la práctica de premasticar alimentos (es decir, masticar alimentos antes de dárselo a un niño), que ocurre sobre todo durante el destete, se ha descrito en varias partes del mundo; sin embargo, la transmisión de la infección por VIH no se había asociado a esta práctica hasta el año 2009. Ese año se comunicaron tres casos de infección pediátrica por VIH, en niños previamente negativos para infección por VIH y que recibían la comida premasticada de un adulto que estaba infectado con el virus. En dos casos se describió sangrado oral concurrente en el adulto que premasticaba el alimento. Los análisis filogenéticos confirmaron que dos de los tres niños se infectaron a través de la exposición a alimentos premasticados por un 
cuidador infectado con el VIH. En ellos se llevó a cabo una investigación a fondo para descartar modos de transmisión alternativos (transmisión vertical, lactancia materna, transfusiones, abuso sexual).

Estos casos proporcionan pruebas concluyentes de la asociación de la premasticación y la infección por VIH. Esta nueva vía de transmisión, que no había sido informada previamente, es una posible explicación de casos de transmisión "tardía" del VIH en los lactantes, hasta ahora atribuidas a la lactancia materna ${ }^{2}$.

La premasticación ha sido informada como una forma de transmisión de la infección por VIH a través de la sangre en la saliva y se ha asociado también con la transmisión de otros patógenos. La premasticación de alimentos sólidos parece ser común en algunas culturas y puede ser usado habitualmente para apoyar y complementar la lactancia materna en zonas donde el agua potable y fórmulas maternizadas no son de fácil disponibilidad ${ }^{3}$.

En madres con periodontitis y con falta de higiene dental ${ }^{4}$, la premasticación probablemente resulta en la mezcla de bacterias, sangre y saliva con el alimento masticado ${ }^{5}$. La premasticación se ha relacionado con la transmisión del virus herpes $8^{6}$, virus de Epstein Barr ${ }^{7}$, Helicobacter pylori ${ }^{8}$ y $\mathrm{VIH}^{2}$ de madres infectadas a sus hijos.

Hasta que el riesgo de premasticación y los factores modificables (enfermedad periodontal) se comprendan mejor, se recomienda que el equipo de salud encargado del cuidado de éstos niños indague sobre esta práctica y oriente sobre opciones de alimentación más seguras.

El CDC condujo una encuesta transversal en nueve clínicas en E.U.A. entre diciembre de 2009 y febrero de 2010 . Se encontró que de 154 cuidadores primarios, $31 \%$ de los niños expuestos al VIH $\geq 6$ meses recibieron alimentos premasticados de un cuidador. Los cuidadores más jóvenes informaron tasas significativamente más altas de esta práctica en comparación con los cuidadores ancianos y los de raza negra mayor frecuencia que los cuidadores de otra raza. Aproximadamente $14 \%$ de los cuidadores en E.U.A reportaron premasticación. Las madres biológicas representaron el $79 \%$ de los premasticadores. No hubo diferencias en la incidencia de premasticación según sexo del niño, país de origen del cuidador, nivel de educación y nivel de ingresos. Esta práctica se inició en niños de tan sólo un mes de edad y se abandonó tan tardíamente como a la edad de 36 meses. Las razones más frecuentemente señaladas para la premasticación son: "el niño quiere algunos de los alimentos del cuidador "(64\%), "el cuidador no quiere que el niño se ahogue" (62\%) y "la premasticación se hace en mi familia" $(31 \%)^{9}$.

El antecedente de exposición en la infancia a la premasticación podría ayudar a identificar a cuidadores infectados por el VIH que realicen esta práctica $^{10}$.

Ya que la premasticación es un comportamiento habitual, sobre todo entre ciertas poblaciones raciales/étnicas, los equipos de salud deben educar a la población sobre el riesgo potencial de transmisión de enfermedades, incluyendo el VIH, a través de esta práctica y desaconsejar a los cuidadores infectados por el VIH a utilizarla.

\section{Resumen}

La incorporación del protocolo de prevención de la transmisión vertical (PTV) del virus de inmunodeficiencia humana (VIH) en mujeres embarazadas, ha reducido la transmisión del VIH en niños a menos de 2\%. En Chile, actualmente el diagnóstico de infección por VIH en niños es infrecuente. Es así que frente a un caso, se debe auditar el cumplimiento del PTV y si éste se cumplió en su totalidad, deben revisarse otras vías posibles de transmisión del VIH. Se presenta un caso clínico en que el VIH pudo haber sido transmitido a través del consumo de alimentos premasticados por una persona infectada con el VIH. La premasticación es una vía de transmisión del VIH no informada antes en nuestro medio, siendo una posible explicación de algunos casos de transmisión "tardía" en lactantes, hasta ahora atribuidas a la lactancia materna. Entendiendo que la premasticación es un comportamiento común, se debe educar a la población, especialmente a los cuidadores infectados por el VIH, sobre el riesgo potencial de esta práctica en la transmisión de enfermedades, incluyendo el VIH.

\section{Referencias bibliográficas}

1.- Centers for Disease Control and Prevention (CDC). HIV Surveillance Report, 2009; vol. 21. http://www.cdc.gov/hiv/topics/surveillance/resources/ reports/. February 2011. Accedido: 11 de marzo de 2011.

2.- Gaur A H, Domínguez K L, Kalish M L, Rivera-Hernández D, Donohoe M, Brooks JT, et al. Practice of feeding premasticated food to infants: a potential risk factor for HIV transmission. Pediatrics 2009; 124: 658-66.

3.- DiNubile M J. Premastication: a possible missing link? Clin Infect Dis 2010; 51: 252-3.

4.- González O A, Ebersole J L, Huang C B. Oral infectious diseases: a potential risk factor for HIV virus recrudescence? Oral Dis 2009; 15: 313-27.

5.- Pelto G H, Zhang Y, Habicht J P. Premastication: the second arm of infant and young child feeding for health and survival? Matern Child Nutr 2010; 6: 4-18.

6.- Wojcicki J M. Traditional behavioural practices, the exchange of saliva and HHV-8 transmission in sub-Saharan African populations. Br J Cancer 2003; 89: 2016-7.

7.- $\quad$ Mbulaiteye S M, Walters M, Engels E A, Bakaki P M, Ndugwa C M, Owor A M, et al. High levels of Epstein-Barr virus DNA

in saliva and peripheral blood from Ugandan mother-child pairs. J Infect Dis 2006; 193: 422-6.

8.- Taylor D N, Blaser M J. The epidemiology of Helicobacter pylori infection. Epidemiol Rev 1991; 13: 42-59.

9.- Centers for Disease Control and Prevention (CDC). Premastication of food by caregivers of HIV-exposed children-nine U.S. sites, 2009-2010. MMWR Morb Mortal Wkly Rep 2011; 60: 273-5.

10.- Hafeez S, Salami O, Alvarado M, Maldonado M, Purswani M, Hagmann S. Infant feeding practice of premastication: an anonymous survey among human immunodeficiency virus-infected mothers. Arch Pediatr Adolesc Med 2011; 165: 92-3. 